\title{
Structure and Dynamics of a CheY-Binding Domain of the Chemotaxis Kinase CheA Determined by Nuclear Magnetic Resonance Spectroscopy ${ }^{\dagger, \sharp}$
}

\author{
Megan M. McEvoy, ${ }^{\S}$ D. Ranjith Muhandiram," Lewis E. Kay," and Frederick W. Dahlquist*, \\ Institute of Molecular Biology, University of Oregon, Eugene, Oregon 97403, and Protein Engineering Network Centres of \\ Excellence and Departments of Medical Genetics, Biochemistry, and Chemistry, University of Toronto, \\ Toronto, Ontario, Canada M5S 1A8 \\ Received November 13, 1995; Revised Manuscript Received February 22, $1996^{\otimes}$
}

\begin{abstract}
The Escherichia coli histidine autokinase CheA plays an important role in coupling signals received from membrane-bound receptors to changes in the swimming behavior of the cells in order to respond appropriately to environmental signals. Here we describe the structure of the $14 \mathrm{kDa}$ fragment of the chemotaxis kinase CheA, residues 124-257, which binds to the downstream targets of phosphorylation, the response regulators $\mathrm{CheY}$ and $\mathrm{CheB}$. This protein fragment contains the Che Ybinding domain flanked on each side by regions that correspond to domain linkers in the intact protein. The structure of the domain was determined from 1429 restraints derived from heteronuclear multidimensional NMR experiments. Hybrid distance geometry-dynamical simulated annealing methods were used to calculate a family of structures that satisfy the experimental distance restraints and torsion angle restraints. The root mean square deviation of the 69 ordered residues in the domain is $0.52 \AA$ for the backbone heavy atoms and $0.99 \AA$ for all heavy atoms. The residues that have been implicated as important for CheY binding form a face consisting of several partially buried hydrophobic residues, framed by charged residues. The dynamic properties of this protein fragment were measured and analyzed using both isotropic and anisotropic models of molecular motion. The linker regions are very flexible and disordered, as evidenced by the very different dynamics properties as compared to the CheY-binding domain. The CheY-binding domain of $\mathrm{CheA}$ is structurally similar to the histidine-containing phosphocarrier, HPr, which is a protein involved in the phosphoenolpyruvate:sugar phosphotransferase (PTS) pathway. This structural similarity suggests a possible evolutionary relationship of the PTS and chemotaxis pathways.
\end{abstract}

Motile bacteria have the ability to respond to environmental changes in order to effect net movement toward food sources and away from toxins [see Parkinson (1993) and Stock et al. (1994) for reviews]. The chemotaxis signal transduction pathway connects differing extracellular conditions to changes in swimming behavior. In Escherichia coli, extracellular ligands are bound by membrane-spanning receptors, which transmit this information to cytosolic components of the pathway, likely through conformational changes in the receptor [see Stock et al. (1991) for a review]. The cytosolic components include the histidine autokinase CheA, which changes its rate of autophosphorylation in response to ligand binding events on the extracellular face of chemotactic receptors (Borkovich et al., 1989; Borkovich \& Simon, 1990; Ninfa et al., 1991; Wolfe et al., 1994). After autophosphorylation, the kinase serves as the phosphate donor for the response regulators $\mathrm{CheY}$ and $\mathrm{CheB}$. Phosphorylated $\mathrm{CheY}$ interacts with the components of the

$\dagger$ This work was supported by a National Research Service Award Pre-Doctoral Training Grant from the NIH to M.M.M. and by NIH Grant GM33677 to F.W.D. L.E.K. was supported by funding from the Medical Research Council of Canada. Computer resources were provided in part by funding from NSF Grant STI-9413532.

$¥$ The coordinates for the restrained minimized average of 54 NMRderived structures have been deposited in the Protein Data Bank (filename 1FWP).

* Author to whom correspondence should be addressed.

$\S$ University of Oregon.

"University of Toronto.

${ }^{\otimes}$ Abstract published in Advance ACS Abstracts, April 15, 1996. flagellar motor and causes a switch in direction of rotation of the motor (Bourret et al., 1990; Welch et al., 1993). Phosphorylated CheB increases its methylesterase activity toward the receptors upon phosphorylation, in order to modulate chemotactic response to static conditions (Borkovich et al., 1992).

CheA is organized into five functional domains responsible for phosphotransfer, CheY binding, catalysis, receptor coupling, and CheW coupling. The CheY-binding domain, the focus of this paper, has been demonstrated to bind specifically to CheY in vitro (Swanson et al., 1995; McEvoy et al., 1995) and likely contains all the determinants of CheA necessary for binding to CheY. Overexpression of the fragment containing the CheY-binding domain in E. coli cells causes an inhibition of the chemotaxis response (Morrison \& Parkinson, 1994), as would be expected if the response regulator $\mathrm{CheY}$ was sequestered and could not interact with the flagellar motor.

The autokinase CheA belongs to the "two-component" family of signal transduction systems, defined on the basis of sequence homology and the identity of the phosphorylated amino acid residues [for review, see Parkinson and Kofoid (1989)]. One component of this family, the kinase, uses ATP to autophosphorylate at a histidine residue. The second component, the response regulator, is a substrate of the kinase. Phosphate is transferred from the histidine of the kinase to an aspartate residue in the response regulator. 
Members of this family are involved in various signaling processes including response to osmolarity, temperature, $\mathrm{pH}$, and nutrient concentration. E. coli may contain as many as 50 homologous but distinct two-component systems (Parkinson \& Kofoid, 1989), which require stringent specificity of one component for its partner to ensure that a given signal elicits the appropriate response. In the chemotaxis kinase CheA, the presence of a response regulator-binding domain may aid in providing this specificity.

In order to better understand the ways in which signals are transmitted faithfully through the cell where many homologous components are present, it is important to understand how recognition occurs at the molecular level. Prior to the determination of the global folds of two of the domains of CheA (Zhou et al., 1995; McEvoy et al., 1995) no structural information was available about the kinase components of this family. The high-resolution structure of the CheY-binding domain of CheA reported here will provide the first step toward an understanding of the precise recognition steps that must occur for proper signal transfer.

In addition to structural information, it is crucial to have an understanding of the motional fluctuations occurring on a variety of time scales. It is likely that protein flexibility is intricately linked with recognition processes, and therefore the dynamic properties of this domain in solution may play a considerable role in response regulator recognition. Relaxation measurements provide a powerful means of obtaining information about backbone amide motions at the atomic level and complement the structural information well. The backbone dynamics of CheA124-257 are presented by analysis of inverse-detected ${ }^{1} \mathrm{H}-{ }^{15} \mathrm{~N} 2 \mathrm{D} \mathrm{NMR}^{1}$ spectroscopy and are discussed in context of the structural information.

\section{EXPERIMENTAL PROCEDURES}

NMR Samples. In order to better define the side chain conformations of valine and leucine residues, stereospecific assignments of the methyl groups were made by the biosynthetically directed fractional ${ }^{13} \mathrm{C}$-labeling method outlined by Neri et al. (1989). To obtain fully ${ }^{15} \mathrm{~N}$-labeled, $10 \%{ }^{13} \mathrm{C}$-labeled CheA124-257, protein was expressed from the pTM22 plasmid (Morrison \& Parkinson, 1994) in E. coli strain $\mathrm{K} 38$ growing in minimal media containing $1 \mathrm{~g} / \mathrm{L}$ $\left({ }^{15} \mathrm{NH}_{4}\right)_{2} \mathrm{SO}_{4}$ and $2 \mathrm{~g} / \mathrm{L}\left[{ }^{12} \mathrm{C}\right]$ glucose as the sole nitrogen and carbon sources, respectively. One hour before induction, 0.2 $\mathrm{g} / \mathrm{L}\left[{ }^{13} \mathrm{C}\right]$ glucose was added to the growing medium. Cells were induced at $\mathrm{OD}_{600}=0.5$ with $1 \mathrm{mM}$ isopropyl $\beta$-Dthiogalactopyranoside and growth was continued for $4 \mathrm{~h}$. Isolation of both partially and fully labeled protein was carried out by the standard procedure (McEvoy et al., 1995).

NMR Spectroscopy. All experiments were carried out at $30{ }^{\circ} \mathrm{C}$ using protein concentrations of $1.5-4 \mathrm{mM}$ CheA124257 in $50 \mathrm{mM}$ sodium phosphate at $\mathrm{pH} 6.5,0.02 \%$ sodium azide, and $10 \% \mathrm{D}_{2} \mathrm{O}$.

NOE cross peaks used to obtain distance restraints for structure calculations were obtained from a ${ }^{13} \mathrm{C},{ }^{15} \mathrm{~N}$ NOESYHSQC (Pascal et al., 1994) spectrum with a $150 \mathrm{~ms}$ mixing time on a Varian UNITY $500 \mathrm{MHz}$ spectrometer equipped with a pulsed field gradient unit. Residual water signal in

\footnotetext{
${ }^{1}$ Abbreviations: HSQC, heteronuclear single-quantum correlation; NMR, nuclear magnetic resonance; NOE, nuclear Overhauser effect; NOESY, nuclear Overhauser effect spectroscopy; rms, root mean square.
}

the spectrum was eliminated using the method of $\mathrm{Mu}$ handiram et al. (1993). $\phi$ angle restraints were obtained from a $J$-modulated $\left[{ }^{15} \mathrm{~N},{ }^{1} \mathrm{H}\right] \mathrm{COSY}$ described in McEvoy et al. (1995).

Data for the stereospecific assignments and dynamics measurements were obtained on a General Electric Omega $500 \mathrm{MHz}$ spectrometer equipped with a Nalorac tripleresonance gradient probe. The stereospecific assignments of valine and leucine methyl groups of CheA124-257 were obtained by examination of a ${ }^{13} \mathrm{C}$ HSQC spectrum (Wider \& Wüthrich, 1993) using a ${ }^{15} \mathrm{~N}, 10 \%{ }^{13} \mathrm{C}$-labeled sample of CheA124-257.

Structure Calculations. All structure calculations were performed using X-PLOR version 3.1 (Brünger, 1993) running on SGI Power Challenge computers. Only the structure of the 69 amino acid CheY-binding domain of CheA, residues 159-227, was calculated; the linker regions were not included. Sixty initial structures were generated with approximately 1200 NOE restraints. Ambiguous NOEs were assigned on the basis of the structures generated, and these restraints were added in further rounds of refinement. The MIDAS molecular graphics system (Ferrin et al., 1988) was used to display calculated structures. The final structures were derived from 1429 experimentally derived restraints of which 1380 were distance restraints (593 intraresidue, 344 sequential, 192 medium range, 205 long range, 46 hydrogen bonding) and 49 were dihedral angle restraints. Fifty-four of the 60 initial structures converged with low energy and had no distance restraint violations greater than $0.5 \AA$ or dihedral angle violations greater than $5^{\circ}$. All 54 converged structures were averaged, resulting in a pairwise root mean square deviation of $0.52 \AA$ for the backbone heavy atoms and $0.99 \AA$ for all heavy atoms.

Backbone Dynamics. The spin-lattice relaxation times $\left(T_{1}\right)$, spin-spin relaxation times $\left(T_{2}\right)$, and heteronuclear ${ }^{15} \mathrm{~N}$ $\left\{{ }^{1} \mathrm{H}\right\}$ NOEs were measured by inverse-detected twodimensional NMR methods (Kay et al., 1989; Clore et al., 1990; Barbato et al., 1992). Seven data sets for determining the $T_{1}$ relaxation rates were collected using delays of 28 , 70, 140, 308, 504, 645, and $840 \mathrm{~ms}$. Eight data sets for determining the $T_{2}$ relaxation rates were collected using delays of 8, 23, 46, 69, 99, 122, 153, and $199 \mathrm{~ms}$. Heteronuclear ${ }^{15} \mathrm{~N}\left\{{ }^{1} \mathrm{H}\right\}$ NOEs were measured using the pulse sequence of Barbato et al. (1992). In this set of experiments, one spectrum was acquired with the NOE effect and another spectrum was acquired without the NOE effect for reference.

The relaxation data were analyzed by both isotropic and anisotropic models of molecular motion. Equations and parameters which relate $T_{1}, T_{2}$, and the NOE factors are described in eqs $1-3$ of Kay et al. (1989), where it is assumed that the relaxation of the ${ }^{15} \mathrm{~N}$ nucleus is governed solely by the dipolar coupling with the directly bonded nucleus and by chemical shift anisotropy. In this case the overall tumbling of the molecule is assumed to be isotropic. Errors in the $T_{1}$ and $T_{2}$ data were estimated from the noise level in the spectra (Farrow et al., 1994). With reasonable accuracy, effects of rapid internal motions can be neglected in the calculation of the overall correlation time $\tau_{\mathrm{m}}$, and therefore $\tau_{\mathrm{m}}$ can be calculated from the ratio of $T_{1} / T_{2}$ (Kay et al., 1989; Clore et al., 1990; Barbato et al., 1992). For the isotropic model of motion, the spectral density function is given by (Lipari \& Szabo, 1982) 


$$
J\left(\omega_{i}\right)=S^{2} \tau_{\mathrm{m}} /\left[1+\left(\omega_{i} \tau_{\mathrm{m}}\right)^{2}\right]+\left(1-S^{2}\right) \tau /\left[1+\left(\omega_{i} \tau\right)^{2}\right]
$$

where $1 / \tau=1 / \tau_{\mathrm{m}}+1 / \tau_{\mathrm{e}}, S^{2}$ is the generalized order parameter measuring the degree of spatial restriction of the motion, and $\tau_{\mathrm{e}}$ is the effective correlation time of the rapid internal motions. The dynamics parameters $S^{2}$ and $\tau_{\mathrm{e}}$ were derived from a combination of the $T_{1}, T_{2}$, and NOE data using an average $\tau_{\mathrm{m}}$ by minimizing the function (Barbato et al., 1992; Zhou et al., 1995)

$$
\begin{aligned}
\mathrm{f}\left(S^{2}, \tau_{\mathrm{e}}\right)= & {\left[\left(T_{1, \text { calc }}-T_{1, \text { meas }}\right) / T_{1, \text { calc }}\right]^{2}+\left[\left(T_{2, \text { calc }}-\right.\right.} \\
& \left.\left.T_{2, \text { meas }}\right) / T_{2, \text { calc }}\right]^{2}+\left[\mathrm{NOE}_{\text {meas }}-\mathrm{NOE}_{\text {calc }}\right]^{2} / 4
\end{aligned}
$$

where the subscripts meas and calc refer to the measured and calculated values, respectively. In this equation, the contribution from the NOE data was decreased by a factor of 4 to account for the larger experimental error in these data.

For the case of anisotropic molecular motion, calculations were performed essentially as described in Lipari and Szabo (1982a,b) and Zhou et al. (1996). Only the residues in secondary structure regions of the CheY-binding domain of CheA that were well resolved were used in the anisotropic calculations. The generalized order parameter $S^{2}$ was calculated by minimizing eq 2 using the form of the spectral density function given by (Lipari \& Szabo, 1982b)

$$
\begin{aligned}
& J(\omega)=A\left\{S^{2} \tau_{1} /\left[1+\left(\omega \tau_{1}\right)^{2}\right]+\left(1-S^{2}\right) \tau_{1 \mathrm{e}} /\right. \\
& \left.\left[1+\left(\omega \tau_{1 \mathrm{e}}\right)^{2}\right]\right\}+(1-A)\left\{S^{2} \tau_{2} /\left[1+\left(\omega \tau_{2}\right)^{2}\right]+\right. \\
& \left.\left(1-S^{2}\right) \tau_{2 \mathrm{e}} /\left[1+\left(\omega \tau_{2 \mathrm{e}}\right)^{2}\right]\right\}
\end{aligned}
$$

where $1 / \tau_{1 \mathrm{e}}=1 / \tau_{1}+1 / \tau_{\mathrm{e}}$ and $1 / \tau_{2 \mathrm{e}}=1 / \tau_{2}+1 / \tau_{\mathrm{e}}$. The local parameter $A$ represents the weight of the two modes of rotation. The adjustable parameters $A, \tau_{1}$, and $\tau_{2}$ can be determined by fitting the relaxation data of a nucleus attached to the backbone of the macromolecule (Lipari \& Szabo, 1982a,b), where $0 \leq A \leq 1$ and for the anisotropic case $\tau_{1}$ $\neq \tau_{2}$. For the case where $\tau_{1}=\tau_{2}$, the result is the same as in the isotropic model of molecular motion (Lipari \& Szabo, 1982a,b). The values used for the effective correlation time $\tau_{\mathrm{e}}$ were those derived from the isotropic model when $\tau_{\mathrm{e}}>$ $10 \mathrm{ps}$ and set to $10 \mathrm{ps}$ when $\tau_{\mathrm{e}}<10 \mathrm{ps}$, since when internal motions are significantly rapid, their contribution to the relaxation parameters is negligible (Lipari \& Szabo, 1982a,b; Clore et al., 1990). The parameters $\tau_{1}$ and $\tau_{2}$ were found by a global fit to the spectral density function varied by a $0.2 \mathrm{~ns}$ step size. For each residue, the local parameters $S^{2}$ and $A$ were derived using the best global fit of $\tau_{1}$ and $\tau_{2}$. Estimates of the errors in the $\tau_{1}$ and $\tau_{2}$ fits were made by Monte Carlo simulation and subsequent fitting of the calculated $T_{1}, T_{2}$, and NOE data (Press et al., 1992; Tjandra et al., 1995).

\section{RESULTS AND DISCUSSION}

Structure of the CheY-Binding Domain of CheA. The NMR experiments were conducted on a 139 amino acid fragment of CheA which contains the 69 residue CheYbinding domain (residues 159-227) as well as two unstructured domain linkers (residues 124-158 and 228-257). Structure calculations using hybrid distance geometrydynamical simulated annealing methods (Nilges et al., 1988) were performed using 1429 NMR-derived constraints for the 69 ordered amino acid residues in the CheY-binding domain of CheA. Stereospecific assignments for the six valine and ten leucine residue methyl groups in the CheY-binding domain were obtained using the method of Wider and Wüthrich (1993), and this information was incorporated into the structure calculations. The results of these calculations yield a structure with the overall fold being an "open-faced $\beta$-sandwich" (Richardson, 1981), as suggested by previous studies (McEvoy et al., 1995). Four antiparallel $\beta$-strands form one face of the domain, and the other face consists of two oppositely oriented $\alpha$-helices (Figure 1). The two helices cross at an angle of approximately $30^{\circ}$ over the $\beta$-sheet which has a slight right-handed twist. The domain contains a wellpacked hydrophobic core consisting in part of residues I162, L164, L175, L179, L195, A197, L199, I203, I208, L212, I216, I221, and F223.

The distribution of NOEs and average backbone rms deviations for residues 159-227 are shown in Figure 2. The distribution of NOEs is fairly regular, resulting in generally uniform rms deviations. The C-terminal region has slightly higher backbone rmsd values, which may result from fewer NOEs in this region as compared to the rest of the domain. The other more variable regions in the calculated structures are in the loop before the first helix, residues K168, A169, and G170 where there are few long-range NOEs, and residues T183 and T184 in the loop after the first helix. The residues in the helices generally have fewer long-range NOEs than residues in the $\beta$-strands, but their positions with respect to the sheet are still relatively well constrained.

The geometric properties of the calculated structures were analyzed by the program PROCHECK (Laskowski et al., 1993). The Ramachandran plot for the restrained minimized average of the 54 converged structures is shown in Figure 3 . No disallowed $\phi, \psi$ angles are present in the calculated structures, although three residues are in the "generously" allowed regions of the plot. These residues, R166, D187, and E217, are all in turn regions that are somewhat less precisely defined than regions of regular secondary structure. Experimentally measured $\phi$ angles from $J$-coupling data were included in the input for the structure calculations, though no other restraints for $\phi, \psi$ angles were used. Bond length violations were heavily penalized in the calculation scheme used, so the bond lengths are consistent within $0.05 \AA$ of small molecule values.

The residues implicated in CheY binding are H181, L182, G201, I203, A204, D206, D207, T209, V211, and F214 (McEvoy et al., 1995). All of these residues are partially or mostly exposed to the solvent (Figure 4). The side chain of F214, for example, is fully solvent exposed on the surface of the molecule in all the structures calculated. Only one medium-range NOE was observed to the side chain of F214. Although it is possible that there may be only a few NOEs to the side chain of F214 due to conformational exchange broadening, it is likely that this residue is exposed for CheY binding. The exposure of hydrophobic residues to the solvent in this region may provide a surface for interactions with CheY, with specificity in part provided by the charged residues on the edges of this patch.

Backbone Dynamics. In order to characterize the motional properties of the CheY-binding domain of CheA, the relaxation parameters of the backbone ${ }^{15} \mathrm{~N}$ nuclei were measured using inverse-detected two-dimensional NMR 

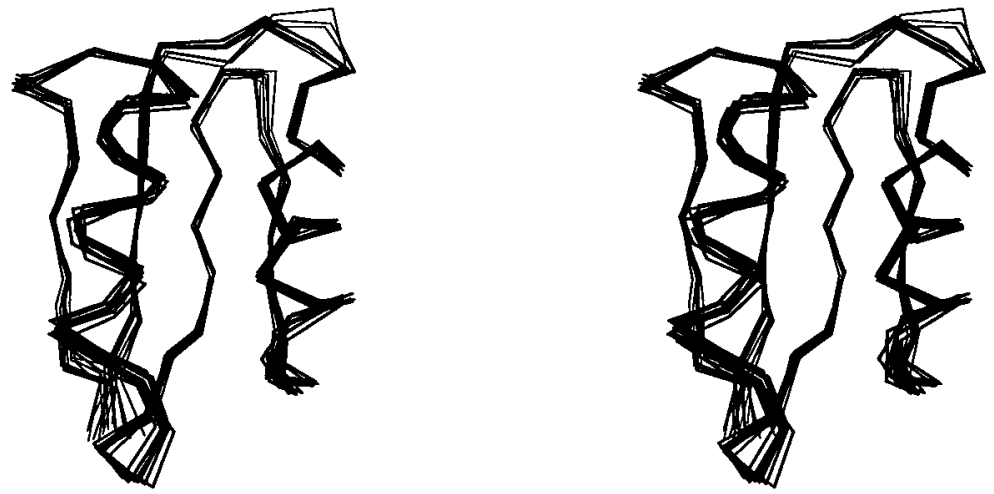

FIGURE 1: Stereoview of the superposition of the $\alpha$-carbon trace of the 10 lowest energy converged structures.
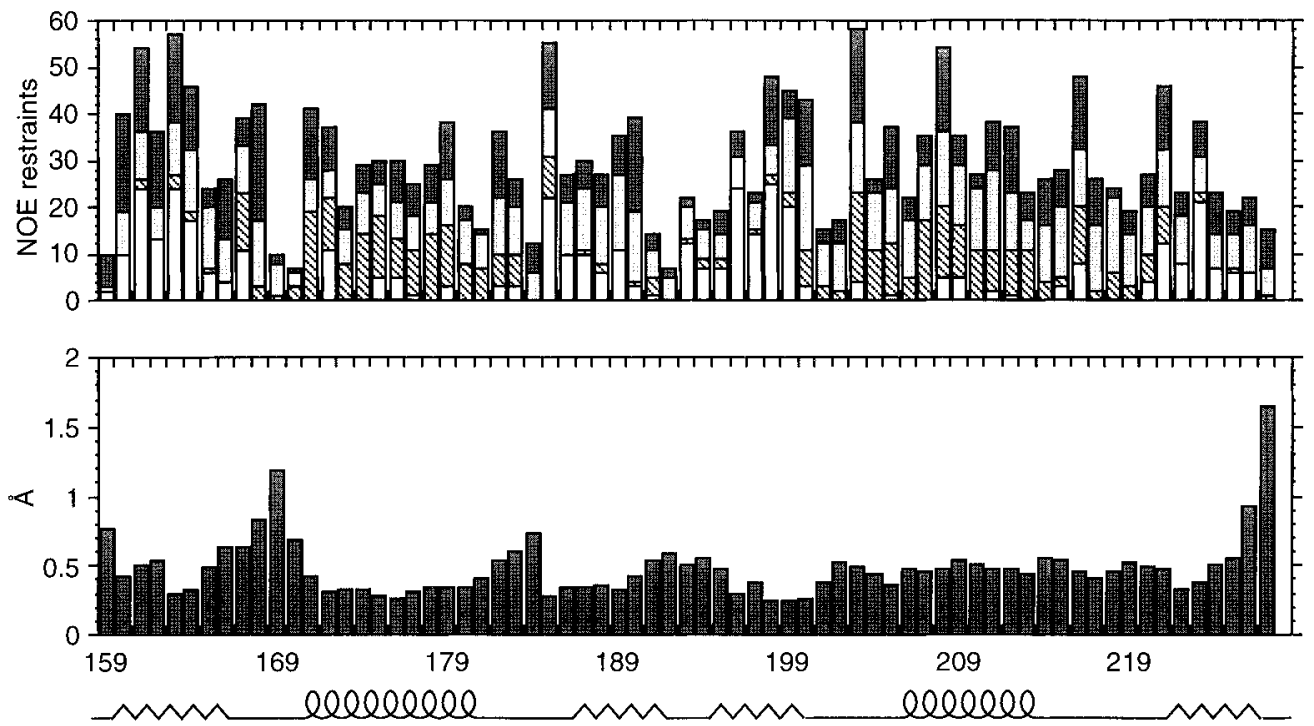

FIGURE 2: (a, top) NOE restraints plotted as a function of the residue number. Black, gray, hatched, and white shading, respectively, indicates intraresidue, sequential, medium-range, and long-range NOEs. (b, bottom) The rms deviation of the backbone $\mathrm{N}$, C $\alpha$, and $\mathrm{C}^{\prime}$ atoms for the average of 54 structures of CheA159-227.

methods. Quantitative data were obtained for 61 wellresolved backbone amide peaks from the CheY-binding domain of CheA124-257. Data were not obtained for the two proline residues, P159 and P200, nor for residues R160, G170, E171, E178, A192, L195, V226, and E227 because of peak overlap or chemical exchange with the solvent. In the linker regions, residues E129, V138, V144, A145, S147, Q150, Q153, S158, S229, V237, L238, A245, and T262 were sufficiently resolved to be amenable to analysis. The other peaks in the domain linkers were overlapped and could not be unambiguously measured.

The ${ }^{15} \mathrm{~N}$ longitudinal relaxation rates $T_{1}$, transverse relaxation rates $T_{2}$, and overall correlation time $\tau_{\mathrm{m}}$ were calculated for each residue listed above (Figure 5). The $T_{1}$ relaxation rates reflect molecular motions occurring on nanosecond and picosecond time scales, whereas the $T_{2}$ relaxation times are sensitive to a combination of motional frequencies ranging from milliseconds to picoseconds. The average longitudinal relaxation value $\left(T_{1}\right)$ for the backbone amides within the CheY-binding domain (residues 159-227) was $564 \pm 52$ ms. The average transverse relaxation value $\left(T_{2}\right)$ within the CheY-binding domain was $112 \pm 13 \mathrm{~ms}$. Relatively large $T_{2}$ values compared to the rest of the values obtained for the CheY-binding domain were obtained for residues T184, L185, T186, G191, L199, D202, I203, A204, and E224 which are primarily located at the ends of secondary structure regions or in the turn regions, suggesting flexibility in these

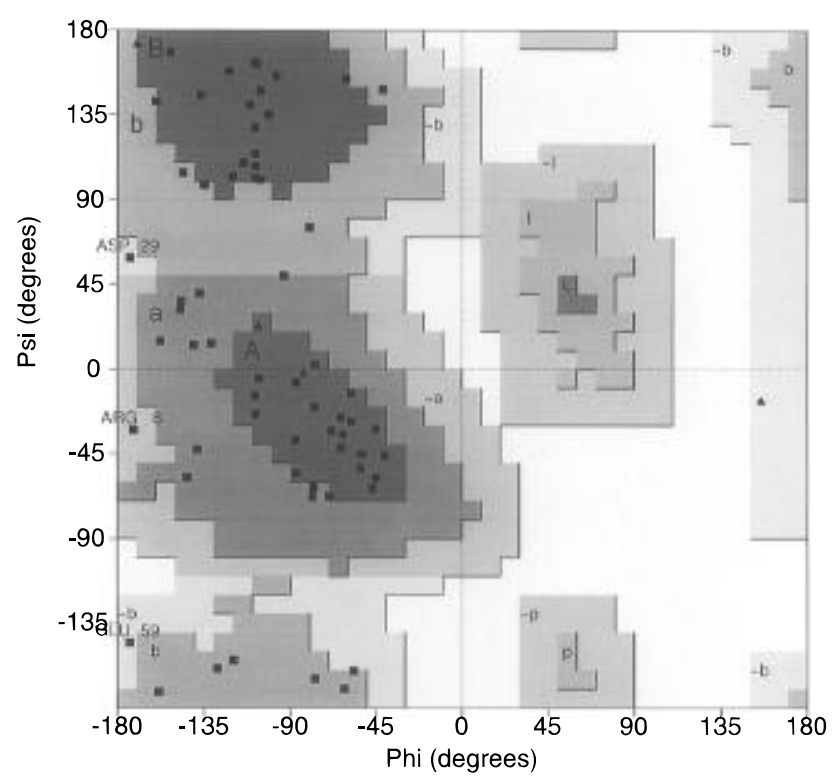

FIGURE 3: Ramachandran plot of the restrained minimized average of 54 converged structures calculated using PROCHECK (Laskowski et al., 1993). Shading from darkest to lightest indicates regions that are most favored, additionally allowed, generously allowed, and disallowed according to the classification of Laskowski et al. (1993). Glycine residues are shown as triangles.

areas. The average $\tau_{\mathrm{m}}$ for the CheY-binding domain was $7.7 \pm 0.8 \mathrm{~ns}$ while the average $\tau_{\mathrm{m}}$ value for the measured 


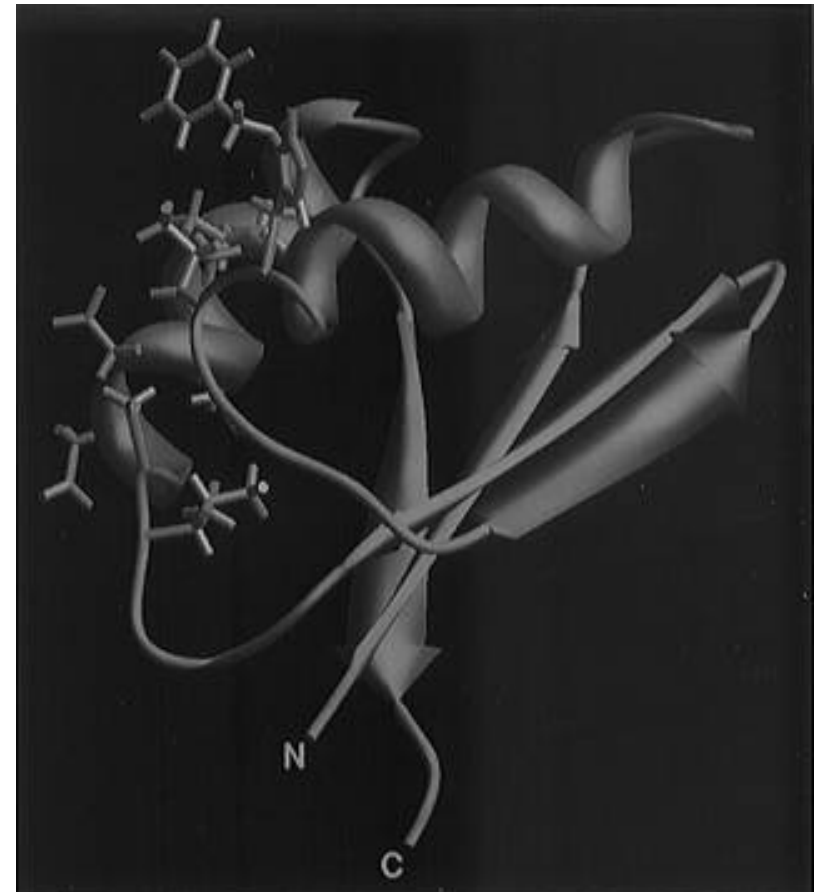

FIGURE 4: Ribbon diagram (Evans, 1993) of the CheY-binding domain of CheA, residues 159-227. The side chains of the residues implicated in CheY binding (McEvoy et al., 1995) are shown in blue.

\section{linker residues was $5.8 \pm 0.6 \mathrm{~ns}$.}

The spectrum of the ${ }^{15} \mathrm{~N}\left\{{ }^{1} \mathrm{H}\right\}$ NOE experiment (Kay et al., 1989) provides an indication of the degree of flexibility of individual amide bond vectors in context of the local structure. The peaks corresponding to residues within the CheY-binding domain of CheA all have positive NOE values in this spectrum, indicating that these residues undergo reorientation with the bulk of the domain (Figure 6a). The linker regions of the protein fragment are highly mobile and disordered as evidenced by the negative peaks for these residues observed in the ${ }^{15} \mathrm{~N}\left\{{ }^{1} \mathrm{H}\right\}$ NOE correlation spectrum (Figure 6b). The peaks for the residues in the linker regions are close to their random coil chemical shift values (McEvoy et al., 1995), which is another indication of a lack of structure. The N-terminal 10 residues of CheA124-257 are part of the phosphotransfer domain of CheA and are observed to be in a helical conformation in the other protein fragments of CheA that have been investigated, CheA1-134 and CheA1-233 (Zhou et al., 1995, 1996). The disorder of these residues in the CheA124-257 construct may result from the lack of elements from the phosphotransfer domain necessary for their structure.

The order parameter $S^{2}$ is an indication of the degree of motion allowed to the amide bond vector. The order parameter $S^{2}$ was calculated using both isotropic and anisotropic models of motion. To a first approximation the isotropic model is adequate for calculation of the relaxation parameters, but for nonspherical molecules, the anisotropic model will provide a more accurate estimation of the molecular motions. The CheY-binding domain is only slightly ellipsoidal, with the longer axis of the ellipsoid running parallel to the helices and perpendicular to the $\beta$-strands of the domain. For the fragment of CheA studied, anisotropy in the overall tumbling is expected, not because of significant nonspherical shape but because of the presence of the domain linkers at the $\mathrm{N}$ - and $\mathrm{C}$-termini of the domain, which may slow the tumbling of the molecule around the axis running perpendicular to the $\beta$-strands.

The overall order parameter $S^{2}$, calculated from the anisotropic model of molecular motion, and the effective correlation time $\tau_{\mathrm{e}}$, calculated from the isotropic model of
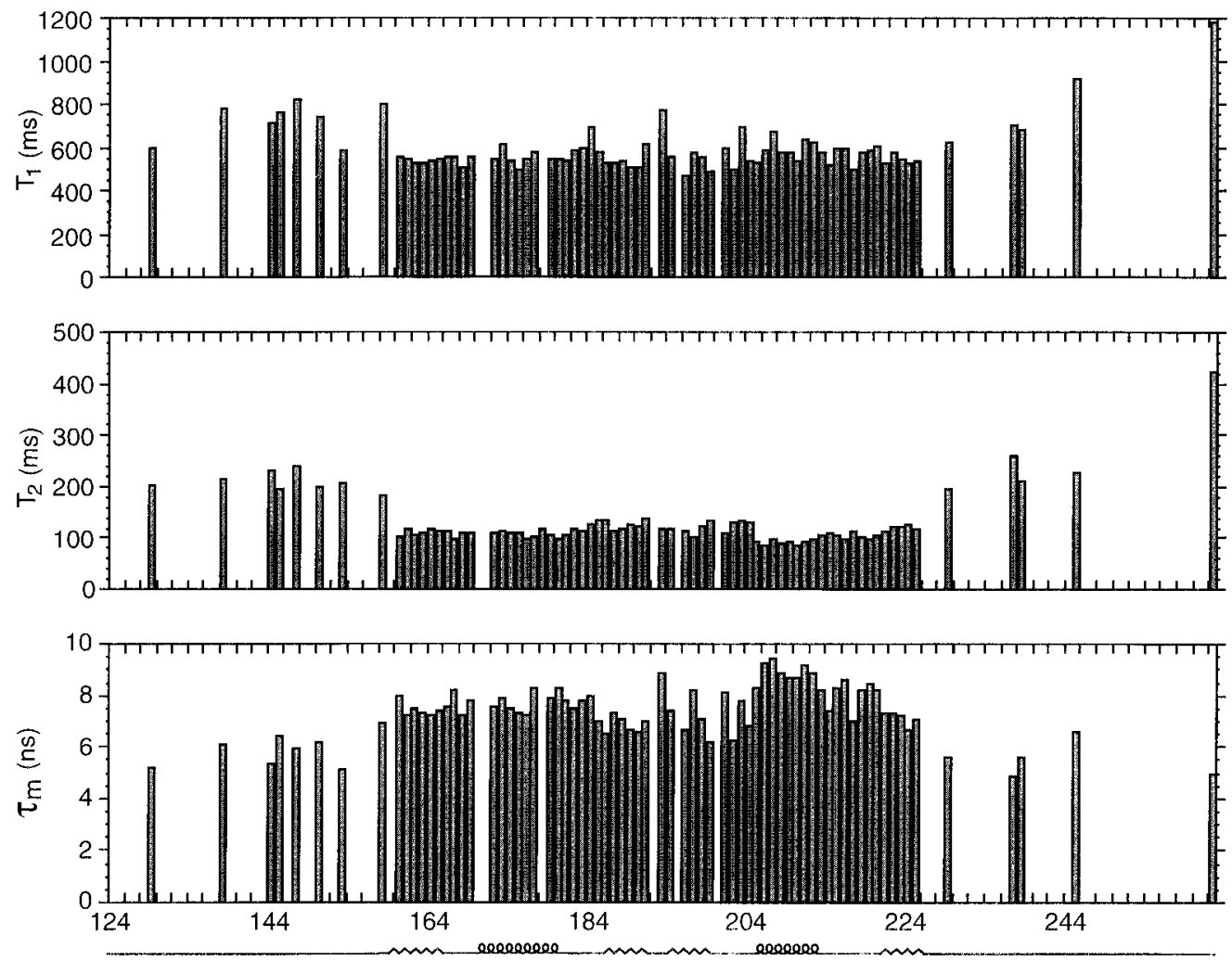

FIGURE 5: Relaxation parameters (a, top) $\mathrm{T}_{1}$ and (b, middle) $T_{2}$ and (c, bottom) $\tau_{\mathrm{m}}$ values plotted as a function of sequence position for the CheA124-257 fragment. 

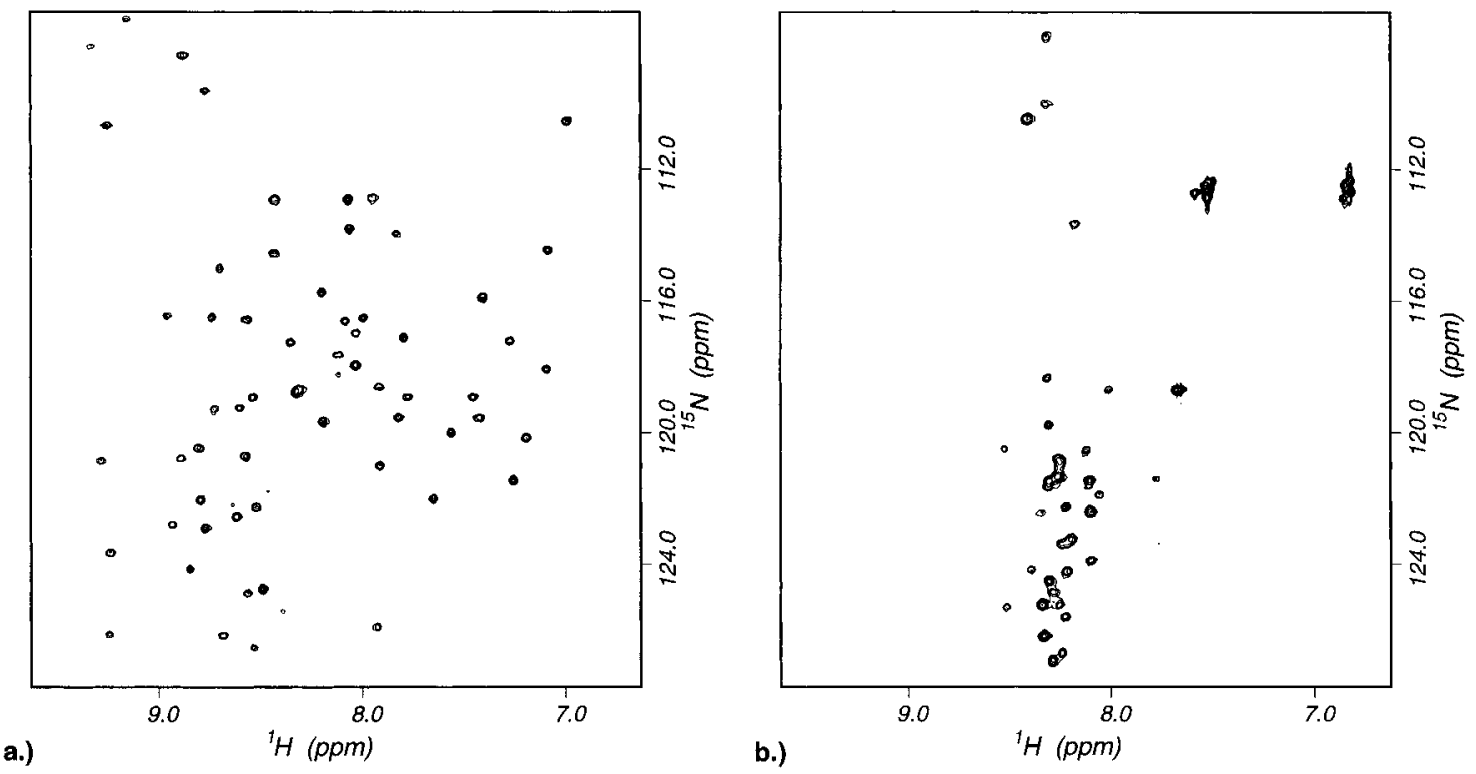

FIGURE 6: ${ }^{15} \mathrm{~N}\left\{{ }^{1} \mathrm{H}\right\}$ NOE spectrum showing (a) positive and (b) negative contours for CheA124-257.
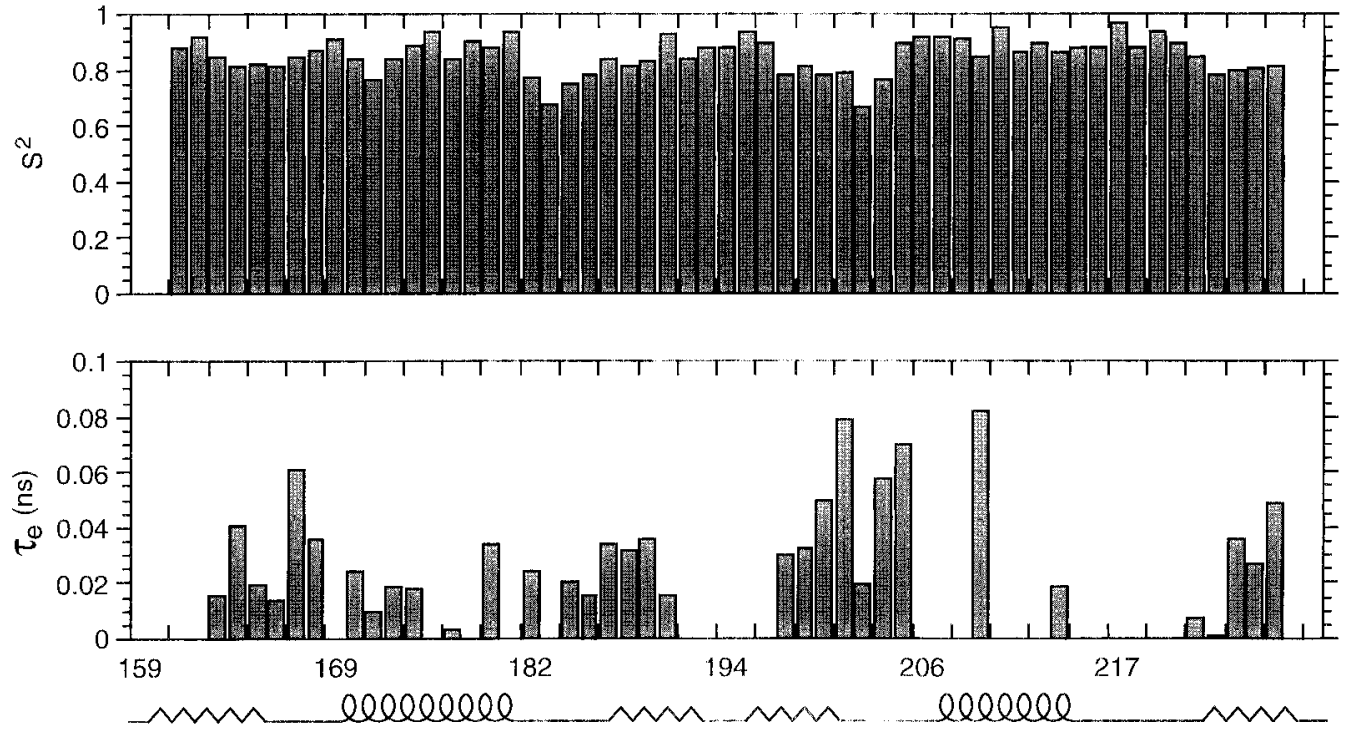

FIGURE 7: (a, top) Overall order parameter $S^{2}$ calculated using the anisotropic model of rotation and (b, bottom) effective correlation time $\tau_{\mathrm{e}}$ calculated using the isotropic model of motion plotted as a function of residue number for the CheY-binding domain of CheA. Where $\tau_{\mathrm{e}}<10 \mathrm{ps}$, the value of $\tau_{\mathrm{e}}$ was set to $10 \mathrm{ps}$ for the calculation of the overall order parameter $S^{2}$, as explained in Experimental Procedures.

molecular motion, are shown in Figure 7 as a function of residue position within the CheY-binding domain of CheA. Because a chemical exchange term was not included in the calculations of the transverse relaxation rate $\left(T_{2}\right)$, only regions of secondary structure were included in the anisotropic order parameter calculations to limit the effects of line-width increases due to chemical exchange processes. The trends seen in the calculations using the isotropic model are similar to those from the anisotropic model, though in general the values of the order parameter $S^{2}$ are lower when calculated using the isotropic model than the anisotropic model (data not shown). The average order parameter is $0.85 \pm 0.06$ for the domain, with a range of values from 0.67 to 0.97 .

The loop regions before and after the two helices differ in overall order parameter values. The loop region after the first helix has order parameter values similar to those of the loop region before the second helix, which are on the same face of the domain. Overall, these order parameter values are lower than the average order parameter for the domain and are lower than the other two loop regions at the other ends of the helices. The loop of residues to the N-terminal side of the first helix and the loop to the C-terminal side of the second helix have relatively high order parameter values. Residues L182, G201, I203, and A204 have been implicated in binding to CheY (McEvoy et al., 1995), and these residues are in the loop regions that have relatively low order parameter values. However, the largest order parameter values are found for residues in the second helix, which also contains many of the residues suggested as important in binding to the response regulator CheY. These data show that generalizations on the rigidity or flexibility of proteinprotein binding surfaces cannot be made, since both the highest and lowest order parameter values are found in regions implicated in $\mathrm{CheY}$ binding. It is possible that a precise combination of rigidity and flexibility is important for high affinity and specificity in protein-protein interactions; alternatively, the dynamics of binding surfaces may play only a minor role in such processes.

Table 1 shows the average $T_{1}, T_{2}, \tau_{\mathrm{m}}$, and $S^{2}$ values for the protein fragment and secondary structures within the 
Table 1: Relaxation Values for CheA124-257 ${ }^{a}$

\begin{tabular}{llccrc}
\hline & $T_{1}(\mathrm{~ms})$ & $T_{2}(\mathrm{~ms})$ & $\tau_{\mathrm{m}}(\mathrm{ns})$ & $S_{\text {iso }}$ & \multicolumn{1}{c}{$S_{\text {aniso }}$} \\
\hline$\langle$ linker $\rangle(13)$ & $762 \pm 156$ & $230 \pm 62$ & $5.8 \pm 0.6$ & $\mathrm{nc}$ & $\mathrm{nc}$ \\
$\langle$ domain $\rangle(56)$ & $564 \pm 52$ & $112 \pm 13$ & $7.7 \pm 0.8$ & $0.82 \pm 0.06$ & $0.85 \pm 0.06$ \\
$\langle\alpha 1\rangle(10)$ (residues 172-182) & $554 \pm 31$ & $107 \pm 8$ & $7.7 \pm 0.4$ & $0.85 \pm 0.05$ & $0.86 \pm 0.06$ \\
$\langle\alpha 2\rangle(9)$ (residues 205-214) & $584 \pm 50$ & $95 \pm 8$ & $8.7 \pm 0.6$ & $0.87 \pm 0.05$ & $0.90 \pm 0.03$ \\
$\langle\alpha 1+\alpha\rangle(19)$ & $566 \pm 42$ & $101 \pm 10$ & $8.2 \pm 0.7$ & $0.86 \pm 0.05$ & $0.88 \pm 0.05$ \\
$\langle\beta 1\rangle(5)$ (residues 161-165) & $538 \pm 11$ & $114 \pm 5$ & $7.4 \pm 0.1$ & $0.83 \pm 0.02$ & $0.86 \pm 0.04$ \\
$\langle\beta 2\rangle$ (4) (residues 187-190) & $520 \pm 15$ & $121 \pm 5$ & $6.9 \pm 0.3$ & $0.83 \pm 0.01$ & $0.85 \pm 0.05$ \\
$\langle\beta 3\rangle$ (4) (residues 194-199) & $545 \pm 37$ & $119 \pm 13$ & $7.2 \pm 0.8$ & $0.82 \pm 0.04$ & $0.84 \pm 0.05$ \\
$\langle\beta 4\rangle(5)$ (residues 221-225) & $542 \pm 19$ & $121 \pm 6$ & $7.1 \pm 0.2$ & $0.81 \pm 0.02$ & $0.81 \pm 0.02$ \\
$\langle\beta 1+\beta 2+\beta 3+\beta 4\rangle(18)$ & $537 \pm 22$ & $119 \pm 7$ & $7.1 \pm 0.4$ & $0.82 \pm 0.02$ & $0.84 \pm 0.04$ \\
\hline
\end{tabular}

${ }^{a}$ The numbers shown are the average values for each of the regions specified. The numbers in parentheses next to the regions are the number of residues in this region used in the calculation. $T_{1}$, longitudinal relaxation rate; $T_{2}$, transverse relaxation rate; $\tau_{\mathrm{m}}$, overall correlation time; $S_{\text {iso }}$, overall order parameter calculated using the isotropic model for rotation; $S^{2}$ aniso, overall order parameter calculated using the anisotropic model for rotation; nc, not calculated.

domain. The relaxation parameters in the domain differ greatly from the linker regions, which is to be expected because the linker is disordered. The relaxation parameters obtained for the helices differ somewhat from the values obtained in the $\beta$-sheet. Kay et al. (1989) found that, for staphylococcal nuclease, there is no correlation between rapid small amplitude motions and secondary structure, in that different secondary structure elements possess similar order parameter values and line widths. In general, slightly smaller average order parameters have been observed for $\beta$-sheets than for helices (Farrow et al., 1994). For the CheY-binding domain of CheA, some of the differences between secondary structure and relaxation rates may be due to orientation of the $\mathrm{H}-\mathrm{N}$ bond vector within the domain as opposed to small amplitude motions. If the domain is considered to be roughly ellipsoidal, the $\mathrm{H}-\mathrm{N}$ bond vectors are oriented parallel to the major and minor axes of the ellipsoid for the helices and strands, respectively. Overall, these differences in order parameters are quite small, and the differences between the elements of secondary structure are even smaller when the calculations are made using the anisotropic model of rotation.

The dynamics of a CheA fragment containing residues $1-233$, consisting of both the phosphotransfer and CheYbinding domains joined by a flexible linker have been studied by fitting to both isotropic and anisotropic models of rotation (Zhou et al., 1996). Though the two domains behave quite independently in this construct, it is clear from the study of Zhou and co-workers that the rotation of the CheY-binding domain is restricted by its connection to the other domain. The flexible linkers in the CheA124-257 construct may restrict reorientation in the same way that attachment to the $\mathrm{N}$-terminal phosphotransfer domain does in the larger CheA $1-233$ construct.

Concluding Remarks. The CheY-binding domain of CheA adopts a fold similar to that seen in a number of other small proteins and domains (McEvoy et al., 1995). One of these proteins, the histidine-containing phosphocarrier HPr, has a role in the phosphoenolpyruvate:sugar phosphotransferase (PTS) pathway in E. coli. Interestingly, chemotaxis to certain sugars involves participation of components of the PTS system. The molecular details of how the environmental information detected by the PTS system flows into the chemotaxis pathway has been unclear. Recently, Lux et al. (1995) have shown that there is a phosphorylation-dependent interaction of the histidine kinase enzyme I of the PTS system with CheA. Enzyme I normally acts to bind and phosphorylate HPr. The structural similarities of the CheY-binding domain of CheA and HPr suggest that enzyme I might interact with the CheY-binding domain of CheA and that this interaction could provide the sensory input from the PTS system. Experiments are underway to test this idea.

The structural similarity of the CheY-binding domain of CheA and HPr, combined with the use of phosphorylated histidine in both systems, suggests an evolutionary relationship between the PTS and chemotaxis systems. As more structures of components of both systems become available, these possible evolutionary relationships should be clarified.

\section{ACKNOWLEDGMENT}

The authors thank B. K. Shoichet, S. J. Remington, and H. Zhou for helpful comments and discussions during the course of this work.

\section{SUPPORTING INFORMATION AVAILABLE}

Table S-1 containing the $T_{1}, T_{2}$, and NOE relaxation data, as well as the calculated values of $\tau_{\mathrm{m}}, \tau_{\mathrm{e}}$, and $S^{2}$ (3 pages). Ordering information is given on any current masthead page.

\section{REFERENCES}

Barbato, G., Ikura, M., Kay, L. E., Pastor, R. W., \& Bax, A. (1992) Biochemistry 31, 5269-5278.

Borkovich, K. A., \& Simon, M. I. (1990) Cell 63, 1339-1348.

Borkovich, K. A., Kaplan, N., Hess, J. F., \& Simon, M. I. (1989) Proc. Natl. Acad. Sci. U.S.A. 86, 1208-1212.

Borkovich, K. A., Alex, L. A., \& Simon, M. I. (1992) Proc. Natl. Acad. Sci. U.S.A. 89, 6756-6760.

Bourret, R. B., Hess, J. F., \& Simon, M. I. (1990) Proc. Natl. Acad. Sci. U.S.A. 87, 41-45.

Brünger, A. (1993) X-PLOR version 3.1: A system for X-ray crystallography and NMR, Yale University Press, New Haven, CT.

Clore, G. M., Driscoll, P. C., Wingfield, P. T., \& Gronenborn, A. M. (1990) Biochemistry 29, 7387-7401.

Evans, S. V. (1993) J. Mol. Graphics 11, 134-138.

Farrow, N. A., Muhandiram, R., Singer, A. U., Pascal, S. M., Kay, C., Gish, G., Shoelson, S. E., Pawson, T., Forman-Kay, J. D., \& Kay, L. E. (1994) Biochemisry 33, 5984-6003.

Ferrin, T. E., Huang, C. C., Jarvis, L. E., \& Langridge, R. (1988) J. Mol. Graphics 6, 13-27.

Kay, L. E., Torchia, D. A., \& Bax, A. (1989) Biochemistry 28, 8972-8979.

Laskowski, R. A., MacArthur, M. W., Moss, D. S., \& Thornton, J. M. (1993) J. Appl. Crystallogr. 26, 283-291.

Lipari, G., \& Szabo, A. (1982a) J. Am. Chem. Soc. 104, 45464559.

Lipari, G., \& Szabo, A. (1982b) J. Am. Chem. Soc. 104, 45594570. 
Lux, R., Jahreis, K., Bettenbrook, K., Parkinson, J. S., \& Lengeler, J. W. (1995) Proc. Natl. Acad. Sci. U.S.A. 92, 11583-11587.

McEvoy, M. M., Zhou, H., Roth, A. R., Lowry, D. F., Morrison, T. B., Kay, L. E., \& Dahlquist, F. W. (1995) Biochemistry 34, 13871-13880.

Morrison, T. B., \& Parkinson, J. S. (1994) Proc. Natl. Acad. Sci. U.S.A. 91, 5485-5489.

Muhandiram, D. R., Farrow, N., Xu, G. Y., Smallcombe, S. J., \& Kay, L. E. (1993) J. Magn. Reson., Ser. B 102, 317-321.

Neri, D., Szyperski, T., Otting, G., Senn, H., \& Wüthrich, K. (1989) Biochemistry 28, 7510-7516.

Nilges, M., Clore, G. M., \& Gronenborn, A. M. (1988) FEBS Lett. 229, 317-324.

Ninfa, E. G., Stock, A., Mowbray, S., \& Stock, J. (1991) J. Biol. Chem. 266, 9764-9770.

Parkinson, J. S. (1993) Cell 73, 857-871.

Parkinson, J. S., \& Kofoid, E. (1992) Annu. Rev. Genet. 26, 71112.

Pascal, S. M., Muhandiram, D. R., Yamazaki, T., Forman-Kay, J., \& Kay, L. E. (1994) J. Magn. Reson., Ser. B 103, 197-201.

Press, W. H., Teukolsky, S. A., Vetterling, W. T., \& Flannery, B. P. (1992) Numerical recipes in FORTRAN: the art of scientific computing, 2nd ed., Cambridge University Press, New York. Richardson, J. S. (1981) Adv. Protein Chem. 34, 167-339.

Stock, J. B., Lukat, G. S., \& Stock, A. M. (1991) Annu. Rev. Biophys. Biophys. Chem. 20, 109-136.

Stock, J., Surette, M., \& Park, P. (1994) Curr. Opin. Neurobiol. 4, 474-480.

Swanson, R., Lowry, D. L., Matsumuara, P., McEvoy, M. M., Simon, M. I., \& Dahlquist, F. W. (1995) Nat. Struct. Biol. 2, 906-910.

Tjandra, M., Kuboniwa, H., Ren, H., \& Bax, A. (1995) Eur. J. Biochem. 230, 1014-1024.

Welch, M., Oosawa, K., Aizawa, S.-I., \& Eisenbach, M. (1993) Proc. Natl. Acad. Sci. U.S.A. 90, 8787-8791.

Wider, G., \& Wüthrich, K. (1993) J. Magn. Reson., Ser. B, 102, 239-241.

Wolfe, A. J., McNamara, B. P., \& Stewart, R. C. (1994) J. Bacteriol. 176, 4483-4491.

Zhou, H., Lowry, D. F., Swanson, R. V., Simon, M. I., \& Dahlquist, F. W. (1995) Biochemistry 34, 13858-13870.

Zhou, H., McEvoy, M. M., Lowry, D. L., Swanson, R. V., Simon, M. I., \& Dahlquist, F. W. (1996) Biochemistry 35, 433-443.

BI952707H 